\title{
Genetic Analysis of TREM2 Variants in Tunisian Patients with Alzheimer's Disease
}

\author{
Zied Landoulsi $^{a}$ Mouna Ben Djebara ${ }^{a, b}$ Imen Kacem ${ }^{a, b}$ Youssef Sidhom ${ }^{a, b}$ \\ Rym Kefi $^{c}$ Sonia Abdelhak ${ }^{c}$ Amina Gargouri-Berrechid ${ }^{a, b}$ Riadh Gouider ${ }^{a, b}$
}

${ }^{a}$ Department of Neurology, UR12SP21, Razi Hospital, Manouba, Tunisia; ${ }^{b}$ Faculty of Medicine of Tunis, University of Tunis El Manar, Tunis, Tunisia; ' Laboratory of Biomedical Genomics and Oncogenetics, Institut Pasteur de Tunis, University of Tunis El Manar, Tunis, Tunisia

\section{Significance of the Study}

- Rare variants within exon 2 of the TREM2 gene increase the risk of Alzheimer's disease in Caucasian populations. This is the first case-control study to assess the association between these variants and the risk of Alzheimer's disease in a North African population. We sequenced exon 2 of the TREM2 gene in a cohort of Tunisian patients with late-onset Alzheimer's disease and healthy individuals and identified 5 variants, none of which was associated with the risk of Alzheimer's disease. Our study does not support a major role for TREM2 in the pathogenesis of late-onset Alzheimer's disease in the Tunisian population.

\section{Keywords}

TREM2 gene · Variants · Alzheimer's disease $\cdot$ North-African population - Case-control study

\section{Abstract \\ Objective: Rare variants in the TREM2 gene have been re- ported to significantly increase the risk of Alzheimer's dis- ease in Caucasian populations. Hitherto, this association was not studied in North African populations. In this work, we aimed to study the association between TREM2 exon 2 vari- ants and the risk of late-onset Alzheimer's disease (LOAD) in a Tunisian population. Subjects and Methods: We se- quenced exon 2 of TREM2 in a Tunisian cohort of 172 LOAD patients and 158 control subjects. We used the Fisher exact test to compare the distribution of allelic frequencies be- tween the two groups. Results: We identified 4 previously reported nonsynonymous variants (p.Asp39Glu, p.Arg62His,}

p.Thr96Lys, and p.Val126Gly) and 1 novel synonymous variant (p.GIn109Gln), none of which was significantly associated with the risk of Alzheimer's disease. Moreover, the rare TREM2 variant (p.Arg47His), which was considered to be a risk factor for Alzheimer's disease in European descent populations, was not detected in our cohort. Conclusion: These findings do not support a major role for TREM2 in the pathogenesis of LOAD in the Tunisian population.

$$
\begin{aligned}
& \text { (c) } 2018 \text { The Author(s) } \\
& \text { Published by S. Karger AG, Basel }
\end{aligned}
$$

\section{Introduction}

TREM2 gene encodes the triggering receptor expressed on myeloid cells 2 , which is highly expressed in microglia of the central nervous system [1] and is involved in regulating the immune system by promoting phagocytosis

\begin{tabular}{ll}
\hline KARGER & $\begin{array}{l}\text { (c) } 2018 \text { The Author(s) } \\
\text { Published by S. Karger AG, Basel }\end{array}$ \\
E-Mail karger@karger.com & $\begin{array}{l}\text { This is an Open Access article licensed under the Creative Commons } \\
\text { Attribution-NonCommercial-4.0 International License (CC BY-NC) } \\
\text { (http://www.karger.com/Services/OpenAccessLicense), applicable to } \\
\text { the online version of the article only. Usage and distribution for com- } \\
\text { mercial purposes requires written permission. }\end{array}$
\end{tabular}

Prof. Riadh Gouider

Service de Neurologie, CHU Razi

1 Rue des Orangers

TN-2010 Manouba (Tunisia)

E-Mail riadh.gouider@gnet.tn 
Table 1. Demographic characteristics of subjects

\begin{tabular}{lccl}
\hline & LOAD patients & Controls & $p$ value \\
\hline Subjects, $n$ & 172 & 158 & \\
Sex ratio (male:female) & $0.5(58: 114)$ & $0.64(62: 96)$ & 0.297 \\
Mean age at examination \pm SD, years & $75.84 \pm 9.64$ & $74.27 \pm 4.17$ & 0.06 \\
Mean age at onset \pm SD, years & $68.69 \pm 10.05$ & & \\
ApoE ع4/ApoE $\varepsilon 4, n(\%)$ & $27(15.7)$ & $3(1.9)$ & $1.310 \mathrm{e}-5$ \\
ApoE $\varepsilon 3 /$ ApoE $\varepsilon 4, n(\%)$ & $73(42.4)$ & $45(28.5)$ & 0.008 \\
ApoE $\varepsilon 3 /$ ApoE $\varepsilon 3, n(\%)$ & $72(41.9)$ & $110(69.6)$ & 0.03 \\
\hline
\end{tabular}

LOAD, late-onset Alzheimer's disease; SD, standard deviation.

and regulating the inflammatory response. The rare missense mutation p.Arg47His (rs75932628) within TREM2 increases the risk of neurodegenerative disorders such as Parkinson's disease [2], essential tremor [3], and late-onset Alzheimer's disease (LOAD). This mutation was identified as a rare risk factor for LOAD in several European descent cohorts with an odds ratio similar to that for apolipoprotein E epsilon 4 (ApoE ع4) $[4,5]$.

TREM2 variants associated with Alzheimer's disease (AD) have been screened in various populations worldwide; however, no studies have been carried-out in North African populations. Most of the aforementioned variants were observed in exon 2 of the TREM2 gene. Therefore, the aim of this work was to evaluate the association of TREM2 exon 2 variants with risk of AD in a sample of the Tunisian population.

\section{Subjects and Methods}

\section{Subjects}

One hundred seventy-two Tunisian patients with LOAD were recruited from the Neurology Department of Razi Hospital, Manouba, Tunisia. Clinical diagnosis of LOAD was done according to the criteria of the National Institute of Neurological and Communication Disorders and Stroke-Alzheimer's Disease and Related Disorders Association (NINCDS-ADRDA) [6]. In addition, 158 unrelated control subjects were recruited from different primary care clinics. None of the control group subjects had cognitive impairment or personal or familial history of neurological and psychiatric disorders. Written informed consent was obtained from each individual prior to enrollment in the genetic study. Research protocols were approved by the Medical Ethics Committee of Razi Hospital and conformed to the guidelines of the Declaration of Helsinki.

\section{ApoE Genotyping and TREM2 Sequencing}

Blood samples were collected from LOAD patients and controls. Genomic DNA was extracted from blood by the salting-out method [7]. ApoE genotyping was performed as previously described [8]. Exon 2 of TREM2 gene was amplified by polymerase chain reaction (PCR) from genomic DNA using two primers: $5^{\prime}$-TGAATGAATGTCTCCTCCCCAG- $3^{\prime}$ and $5^{\prime}$-CAGCCACTGCCCACTCA $-3^{\prime}$, under the following reaction conditions: denaturation at $95^{\circ} \mathrm{C}$ for $5 \mathrm{~min}$ followed by 30 cycles of $95^{\circ} \mathrm{C}$ for $30 \mathrm{~s}$, $60^{\circ} \mathrm{C}$ for $30 \mathrm{~s}$, and $72^{\circ} \mathrm{C}$ for $30 \mathrm{~s}$, and a final cycle of 7 -min extension at $72^{\circ} \mathrm{C}$. PCR products were purified and sequenced using the BigDye Terminator Cycle Sequencing Kit v1.1 on a 3,500xl Genetic Analyzer DNA sequencer (Applied Biosystems, Foster City, CA, USA). Sequences were analyzed using the SeqScape software (Applied Biosystems) and compared to the TREM2 GenBank reference sequence (NM_018965).

\section{In silico Tools}

The impact of TREM2 variations was predicted using SIFT (Sorting Intolerant From Tolerant; http://sift.jcvi.org/www/SIFT_ chr_coords_submit.html), PolyPhen-2 (Polymorphism Phenotyping v2; http://genetics.bwh.harvard.edu/pph2/) and PredictSNP2 (http://loschmidt.chemi.muni.cz/predictsnp2/) [9]. Frequencies of variants were reported from exome database $(1,000 \mathrm{G}$; http://browser.1000genomes.org/index.html), ExAC (http://exac. broadinstitute.org/), EVS (http://evs.gs.washington.edu/EVS/), and Great Middle East (GME) Variome Project database (http:// igm.ucsd.edu/gme/data-browser.php) including 508 samples from North Africa.

\section{Statistical Analysis}

We used Fisher's exact test to compare the distribution of allelic frequencies between LOAD patients and control groups. ApoE genotype and sex distributions were compared using the $\chi^{2}$ test and mean age was compared using the $t$ test. All analyses were two-tailed and a $p$ value of 0.05 or less was considered statistically significant. Odds ratios and 95\% confidence intervals were calculated using SPSS.

\section{Results}

Demographic characteristics and ApoE genotypes of Tunisian LOAD patients $(n=172)$ and controls $(n=158)$ are summarized in Table 1. Both groups had similar sex ratios and similar mean ages. ApoE genotyping showed a 
Table 2. Variants of TREM2 after sequencing of exon 2 in LOAD patients and controls

\begin{tabular}{|c|c|c|c|c|c|c|c|c|c|c|c|}
\hline Variant & $\mathrm{db}$ SNP ID & $\begin{array}{l}\text { Position } \\
\text { (GRCh37) }\end{array}$ & $\begin{array}{l}\text { Exonic } \\
\text { function }\end{array}$ & $\begin{array}{l}\text { LOAD patients } \\
\text { (carriers, } \\
\text { MAF) }\end{array}$ & $\begin{array}{l}\text { Controls } \\
\text { (carriers, } \\
\text { MAF) }\end{array}$ & $\begin{array}{l}p \\
\text { value }\end{array}$ & $\begin{array}{l}\text { OR } \\
(95 \% \mathrm{CI})\end{array}$ & \multicolumn{4}{|c|}{ Population frequency data } \\
\hline p.Arg62His & rs143332484 & g.41129207C>T & nonsyn & $2(0.011)$ & 0 & 0.49 & NA & 0.005 & 0.008 & 0.007 & 0 \\
\hline p.Thr96Lys & rs 2234253 & g.41129105G>T & nonsyn & $5(0.029)$ & $6(0.037)$ & 0.76 & $\begin{array}{l}1.3 \\
(0.39-4.36)\end{array}$ & 0.041 & 0.012 & 0.039 & 0 \\
\hline
\end{tabular}

LOAD, late-onset Alzheimer's disease; db SNP, single nucleotide polymorphism database; MAF, minor allele frequency; syn, synonymous; nonsym, nonsynonymous.

Table 3. Characteristics of individuals carrying the TREM2 variants

\begin{tabular}{|c|c|c|c|c|c|}
\hline Variant & Carrier & Gender & $\begin{array}{l}\text { Age at examination/ } \\
\text { Age at onset, } \\
\text { years }\end{array}$ & $\begin{array}{l}\text { ApoE } \\
\text { genotype }\end{array}$ & $\begin{array}{l}\text { Family history of } \\
\text { neurodegenerative } \\
\text { disease }\end{array}$ \\
\hline p.Asp39Glu & patient 1 & $\mathrm{~F}$ & $86 / 82$ & $\varepsilon 4 / \varepsilon 4$ & yes \\
\hline \multirow{2}{*}{ p.Arg62His } & patient 2 & $\mathrm{M}$ & $79 / 71$ & $\varepsilon 3 / \varepsilon 3$ & yes \\
\hline & patient 3 & $\mathrm{~F}$ & $78 / 71$ & $\varepsilon 3 / \varepsilon 3$ & yes \\
\hline \multirow[t]{10}{*}{ p.Thr96Lys } & patient 4 & $\mathrm{M}$ & $79 / 66$ & $\varepsilon 4 / \varepsilon 4$ & yes \\
\hline & patient 5 & $\mathrm{M}$ & $69 / 67$ & $\varepsilon 3 / \varepsilon 3$ & yes \\
\hline & patient 6 & $\mathrm{~F}$ & $72 / 65$ & $\varepsilon 3 / \varepsilon 4$ & yes \\
\hline & patient 7 & $\mathrm{~F}$ & $80 / 75$ & $\varepsilon 3 / \varepsilon 4$ & yes \\
\hline & patient 8 & $\mathrm{~F}$ & $89 / 79$ & $\varepsilon 3 / \varepsilon 3$ & yes \\
\hline & control 1 & $\mathrm{M}$ & $71 /-$ & $\varepsilon 3 / \varepsilon 3$ & no \\
\hline & control 2 & M & $68 /-$ & $\varepsilon 3 / \varepsilon 4$ & no \\
\hline & control 3 & $\mathrm{~F}$ & $70 /-$ & $\varepsilon 3 / \varepsilon 3$ & no \\
\hline & control 4 & $\mathrm{M}$ & $68 /-$ & $\varepsilon 3 / \varepsilon 3$ & no \\
\hline & control 5 & $\mathrm{~F}$ & $70 /-$ & $\varepsilon 3 / \varepsilon 4$ & no \\
\hline \multirow[t]{5}{*}{ p.Gln109Gln } & patient 9 & $\mathrm{M}$ & $85 / 70$ & $\varepsilon 3 / \varepsilon 4$ & yes \\
\hline & patient 10 & $\mathrm{~F}$ & $75 / 65$ & $\varepsilon 4 / \varepsilon 4$ & yes \\
\hline & patient 11 & $\mathrm{~F}$ & $72 / 67$ & $\varepsilon 3 / \varepsilon 3$ & no \\
\hline & patient 12 & $\mathrm{~F}$ & $85 / 68$ & $\varepsilon 3 / \varepsilon 4$ & no \\
\hline & patient 13 & $\mathrm{~F}$ & $77 / 72$ & $\varepsilon 3 / \varepsilon 3$ & no \\
\hline p.Val126Gly & patient 14 & $\mathrm{M}$ & $82 / 72$ & $\varepsilon 3 / \varepsilon 4$ & yes \\
\hline
\end{tabular}

significant difference in genotype distributions between the two groups; as expected, the ApoE $\varepsilon 4$ allele was overrepresented in LOAD patients compared to the control group ( 36.9 vs. $16.1 \% ; p<0.05$ ). The ApoE $\varepsilon 2$ allele was not detected in patients and controls.

Sequencing of exon 2 of the TREM2 gene revealed 5 variants in $14 \mathrm{LOAD}$ patients and 1 variant in 5 controls. As shown in Table 2, 4 previously reported nonsynonymous variants (p.Asp39Glu, p.Arg62His, p.Thr96Lys, and p.Val126Gly) and 1 novel synonymous variant (p. Gln109Gln) were observed in patients. The variant $p$. Thr96Lys was also identified in controls. All variants were present in the heterozygous state and were detected in separate individuals (no individuals carried 2 or more variants). Among the 14 patients carrying the TREM2 variants, 11 had a positive family history of AD (Table 3 ).

The frequencies of each variation in the population's exome sequencing database are detailed in Table 2. No common variants having minor allele frequency (MAF) $>5 \%$ were found, and 2 variants (p.Val126Gly and p.Asp$39 \mathrm{Glu}$ ) were rare, with $\mathrm{MAF}<1 \%$. Three variants were predicted to have a probable damaging effect (p.Asp39Glu, p.Thr96Lys, and p.Val126Gly; Table 4). The association analysis of all the identified TREM2 variants and $\mathrm{AD}$ 
Table 4. In silico prediction of TREM2 missense variants

\begin{tabular}{llllll}
\hline Variant & db SNP ID & Position $(G R C h 37)$ & SIFT & Polyphen2 & PredictSNP2 \\
\hline p.Asp39Glu & rs200392967 & g.41129275G $>$ C & tolerated & possibly damaging & deleterious \\
p.Arg62His & rs143332484 & g.41129207C $>$ T & tolerated & benign & neutral \\
p.Thr96Lys & rs2234253 & g.41129105G $>$ T & damaging & probably damaging & neutral \\
p.Val126Gly & rs121908402 & g.41129015A $>$ C & damaging & Probably damaging & deleterious \\
\hline
\end{tabular}

Table 5. Summary of all variant screening studies of TREM2 in patients with Alzheimer's disease (AD)

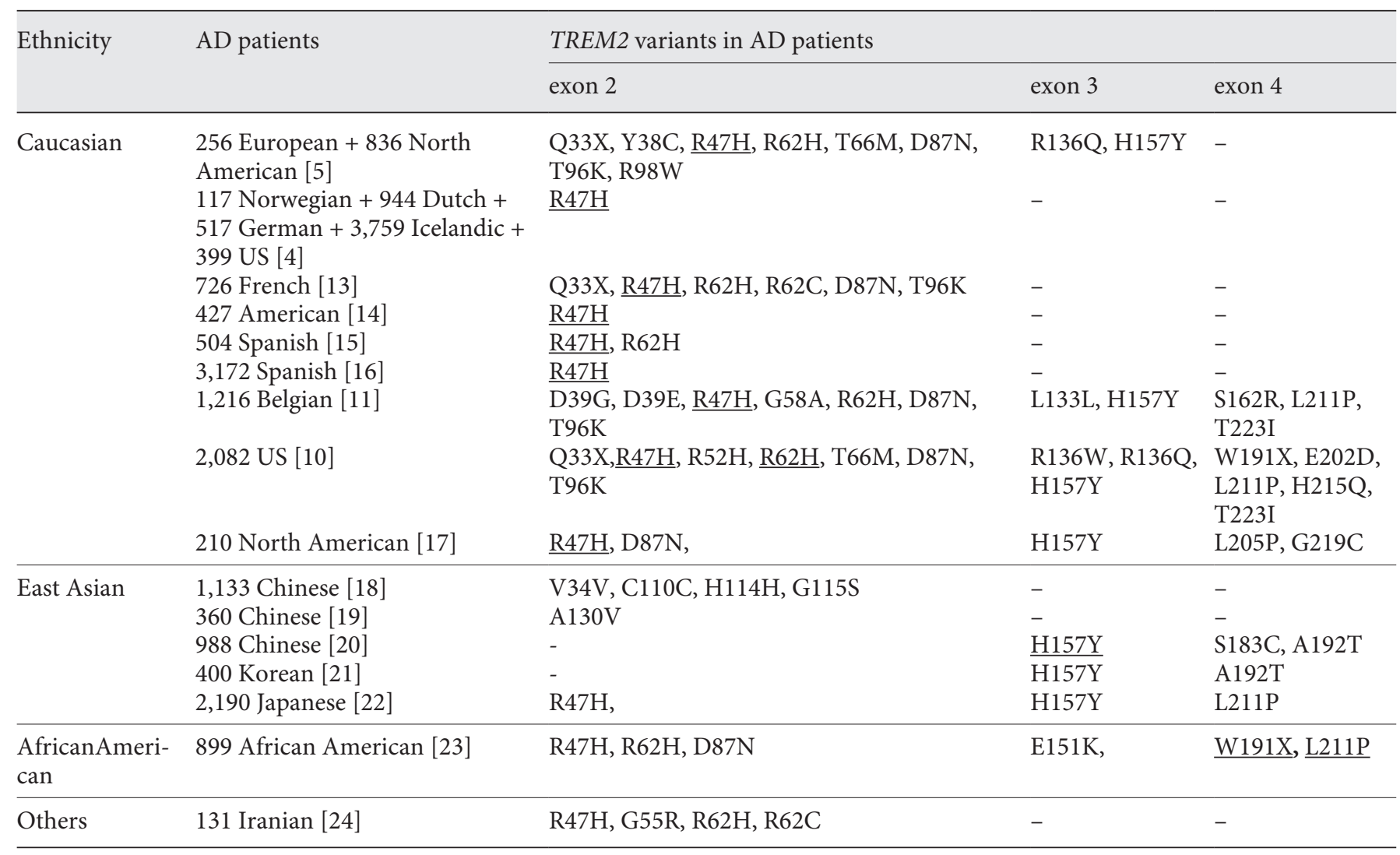

Variations significantly associated with AD are underlined.

was not statistically significant (Table 2). Even the rare variants p.Val126Gly and p.Asp39Glu were not significantly associated with $\mathrm{AD}(p>0.05)$.

\section{Discussion}

This is the first investigation into the possible association between TREM2 variants and risk of $\mathrm{AD}$ in a North African population. In this study, we sequenced exon 2 of the TREM2 gene in a cohort of Tunisian patients with
LOAD and healthy individuals, and identified 5 variants (p.Asp39Glu, p.Arg62His, p.Thr96Lys, p.Gln109Gln, and p.Val126Gly). The p.Arg47His mutation was not found in our cohort. Association analysis revealed that none of the identified variants were associated with $\mathrm{AD}$ risk. Therefore, we could not test the interaction of the ApoE $\varepsilon 4$ allele with these variants.

A summary of the reported screening studies for TREM2 mutations to date, including AD patients, is presented in Table 5. TREM2 p.Arg62His was previously observed in $\mathrm{AD}$ patients and controls in Spanish, Belgian, 
and French populations (Table 5), and did not show a nominally significant association with $\mathrm{AD}$. However, a previous case-control study of large European American descent cohorts showed that in addition to p.Arg47His [10], p.Arg62His is a risk factor for AD. The variant rs2234253 (p.Thr96Lys), detected in Belgian, French, and Japanese populations (Table 5), was not shown to enhance $\mathrm{AD}$ risk. In our study, p.Thr96Lys was the unique variant detected in controls and LOAD patients with equivalent MAF. According to public exome databases, this variant was more common in African populations $(\mathrm{MAF}=14.5 \%, 1,000 \mathrm{G})$ than other populations, but was not present in North African populations of the GME (Table 2).

Two rare variants predicted to be deleterious, p.Asp39Glu and p.Val126Gly, were identified in our study. The p.Asp39Glu was previously reported in $2 \mathrm{AD}$ patients at the heterozygous state [11], while homozygous p.Val$126 \mathrm{Gly}$ was reported in 2 patients with Nasu-Hakola disease [12]. Among the 5 variants, variant Gln109Gln was not found in any of the exome databases. However, this variant is synonymous and does not change the amino acid sequence of TREM2 protein.

The previously reported rare variant rs75932628 (p. Arg47His), identified to be a risk factor for AD in Caucasian populations (Table 5), was not found in our cohort. Our findings are in agreement with published reports of African American, Iranian, and East Asian populations (Table 5) which showed that the p.Arg47His mutation could not be linked to an increased risk of AD. In our study, the lack of association of the p.Arg47His variant may be due to its very low MAF in the Tunisian population. In the GME database, this genetic polymorphism had a global MAF of 0.15 and $0.1 \%$ in North African pop- ulations. Moreover, this variant is considered to be a risk factor according to the ethnicity of the population. AD risk in the Tunisian population may be influenced by genetic and/or environmental factors which might reduce the effect of this variant. However, the limitation of our work is the small cohort size, which could explain the lack of statistical significance.

\section{Conclusion}

This study is the first to explore the possibility of an association of TREM2 with the risk of AD in North Africa, particularly in Tunisia. This population seems to be closer to the African American or East Asian populations than to the European ones regarding the AD risk factor p.Arg47His. We hypothesize that variations in exon 2 of TREM2 may not play a major role in the pathogenesis of LOAD in the Tunisian population. However, further studies on larger cohorts of North African populations are needed to confirm this hypothesis.

\section{Acknowledgements}

We are grateful to all the patients and their family members for participating in this study. This research was supported by grants from the Tunisian Ministry of Higher Education and Scientific Research and the Tunisian Ministry of Health.

\section{Disclosure Statement}

The authors declare that they have no conflicts of interest.

\section{References}

1 Colonna M, Wang Y: TREM2 variants: new keys to decipher Alzheimer disease pathogenesis. Nat Rev Neurosci 2016;17:201-207.

2 Rayaprolu S, Mullen B, Baker M, et al: TREM2 in neurodegeneration: evidence for association of the p.R47H variant with frontotemporal dementia and Parkinson's disease. Mol Neurodegener 2013;8:19.

3 Ortega-Cubero S, Lorenzo-Betancor O, Lorenzo E, et al: TREM2 $\mathrm{R} 47 \mathrm{H}$ variant and risk of essential tremor: a cross-sectional international multicenter study. Parkinsonism Relat Disord 2015;21:306-309.

4 Jonsson T, Stefansson H, Steinberg S, et al: Variant of TREM2 associated with the risk of Alzheimer's disease. N Engl J Med 2013;368: 107-116.
5 Guerreiro R, Wojtas A, Bras J, et al: TREM2 variants in Alzheimer's disease. N Engl J Med 2013;368:117-127.

6 McKhann G, Drachman D, Folstein M, et al: Clinical diagnosis of Alzheimer's disease: report of the NINCDS-ADRDA Work Group under the auspices of Department of Health and Human Services Task Force on Alzheimer's Disease. Neurology 1984;34:939-944.

7 Miller SA, Dykes DD, Polesky HF: A simple salting out procedure for extracting DNA from human nucleated cells. Nucleic Acids Res 1988; 16:1215.

8 Hixson JE, Vernier DT: Restriction isotyping of human apolipoprotein E by gene amplification and cleavage with HhaI. J Lipid Res 1990; 31:545-548.
9 Bendl J, Musil M, Stourac J, et al: PredictSNP2: a unified platform for accurately evaluating SNP effects by exploiting the different characteristics of variants in distinct genomic regions. PLoS Comput Biol 2016;12:e1004962.

10 Jin SC, Benitez BA, Karch CM, et al: Coding variants in TREM2 increase risk for Alzheimer's disease. Hum Mol Genet 2014;23:58385846.

11 Cuyvers E, Bettens K, Philtjens S, et al: Investigating the role of rare heterozygous TREM2 variants in Alzheimer's disease and frontotemporal dementia. Neurobiol Aging 2014; 35:726.e711-e729. 
12 Klunemann HH, Ridha BH, Magy L, et al: The genetic causes of basal ganglia calcification, dementia, and bone cysts: DAP12 and TREM2. Neurology 2005;64:1502-1507.

13 Pottier C, Wallon D, Rousseau S, et al: TREM2 $\mathrm{R} 47 \mathrm{H}$ variant as a risk factor for early-onset Alzheimer's disease. J Alzheimers Dis 2013; 35:45-49.

14 Gonzalez Murcia JD, Schmutz C, Munger C, et al: Assessment of TREM2 rs75932628 association with Alzheimer's disease in a population-based sample: the Cache County Study. Neurobiol Aging 2013;34:2889.e2811-e2883.

15 Benitez BA, Cooper B, Pastor P, et al: TREM2 is associated with the risk of Alzheimer's disease in Spanish population. Neurobiol Aging 2013;34:1711.e1715-e1717.

16 Ruiz A, Dols-Icardo O, Bullido MJ, et al: Assessing the role of the TREM2 $\mathrm{p}$. R47H variant as a risk factor for Alzheimer's disease and frontotemporal dementia. Neurobiol Aging 2014;35:444.e441-e444.
17 Ghani M, Sato C, Kakhki EG, et al: Mutation analysis of the MS4A and TREM gene clusters in a case-control Alzheimer's disease data set. Neurobiol Aging 2016;42:217.e217-217.e213.

$18 \mathrm{Yu} \mathrm{JT}$, Jiang T, Wang YL, et al: Triggering receptor expressed on myeloid cells 2 variant is rare in late-onset Alzheimer's disease in Han Chinese individuals. Neurobiol Aging 2014; 35:937.e931-e933.

19 Jiao B, Liu X, Tang B, et al: Investigation of TREM2, PLD3, and UNC5C variants in patients with Alzheimer's disease from mainland China. Neurobiol Aging 2014;35:2422. e9-2422.e11.

20 Jiang T, Tan L, Chen Q, et al: A rare coding variant in TREM2 increases risk for Alzheimer's disease in Han Chinese. Neurobiol Aging 2016;42:217.e211-e213.
21 Chung SJ, Kim MJ, Kim J, et al: Exome array study did not identify novel variants in $\mathrm{Al}$ zheimer's disease. Neurobiol Aging 2014;35: 1958.e1913-e1954.

22 Miyashita A, Wen Y, Kitamura N, et al: Lack of genetic association between TREM2 and late-onset Alzheimer's disease in a Japanese population. J Alzheimers Dis 2014;41:10311038

23 Jin SC, Carrasquillo MM, Benitez BA, et al: TREM2 is associated with increased risk for Alzheimer's disease in African Americans. Mol Neurodegener 2015;10:19.

24 Mehrjoo Z, Najmabadi A, Abedini SS, et al: Association study of the TREM2 gene and identification of a novel variant in exon 2 in Iranian Patients with late-onset Alzheimer's disease. Med Princ Pract 2015;24:351-354. 